\title{
Research on the Gap Flow Simulation of Debris Removal Process for Small Hole EDM Machining with Ti Alloy
}

\author{
Wenchao Zhang ${ }^{a}$, Yu Liu ${ }^{b, *}$, Shengfang Zhang ${ }^{c}$, Fujian Ma ${ }^{d}$, Puyong \\ Wang ${ }^{\mathrm{e}}$ and Changgang Yan
}

\author{
School of Mechanical Engineering, Dalian Jiaotong University, Dalian 116028, China. \\ achaochao_0627@163.com, ' liuyu_ly12@126.com, 'czf@djtu.edu.cn, \\ dmafujianyx@163.com, ${ }^{\mathrm{e}} 523251918 @ q q . c o m,{ }^{\mathrm{f}} \mathrm{ycg} @$ djtu.edu.cn
}

Keywords: EDM; Small holes machining; Gap flow field; Debris

\begin{abstract}
In EDM, especially in small holes EDM machining, there will be a lot of debris produced which is difficult to remove from narrow gap. If the debris can't be removed in time, it will affect the machining accuracy and efficiency, and sometimes even unable to complete the processing. In this paper, the debris movement and escaping process in gap flow between electrodes in the case of Ti alloy small hole EDM machining were studied by combining the means of simulation analysis and experiment study. The perturbation generated by the movement of self-adaptive tool in the gap flow were taken into consideration and the methods of smoothing and remeshing were also applied in order to build a model for the debris movement in the gap flow. And then, the law of debris movement under different depths was analyzed and the distribution rules about pressure field and velocity vector were summarized. Finally, the model was verified by the experiment result showing that the appearances of uneven wear at the bottom of hole and the taper wall are both in accordance with simulating conclusions.
\end{abstract}

\section{Introduction}

Because of high specific strength and extraordinary corrosion resistance, Titanium (Ti) alloys are widely used in the field of aerospace, medical devices and ocean development nowadays. However, such alloys have very high tensile strength, toughness ${ }^{[1]}$ and low thermal conductivity, all these factors bring about low efficiency, high tool wear along with poor machining accuracy and finish surface in machining small holes with conventional methods like drilling. Sometimes, it is even unable to achieve the machining in the cases of small deep hole machining.

The principle of electrical discharge machining (EDM) is to use the eroding effect of controlled electric spark discharges on the electrodes to remove excess material. As there is no mechanical contact/force between the tool and workpiece during the whole process, it is thus especially recommended to manufacture small features on difficult-to-cut materials like Ti alloys. However, there are also some problems during the operation of machining small holes on Ti alloys by EDM. As a lot of debris is generated in the process, if the debris stays in the gap and can not be washed away in time, the electrical conductivity of the dielectric increases, leading to a bad control of the process and poor machining quality ${ }^{[2]}$. For this reason, flushing is often used. Flushing is the most important function in EDM operation, it is a process that introduces clean filtered dielectric fluid into the spark gap. However, in small hole EDM machining, the gap is so narrow that the flushing does not act well, as a result, a lot of debris will remain in the machining region influencing the EDM process, the matters get worse when a deeper hole is machined.

Jia Z. simulated the flow field with Fluent software showing that the distribution of inter-electrode discharge debris is only related to the debris size. The motion of dielectric liquid is in accordance with inter-electrode flow law ${ }^{[3]}$. Chu Z. conducted experiments to analyze flow field, motion and concentration of debris after electrode jumped and concluded that the speed and cycle of the tool were two significant factors which influence the performance of EDM, and high speed motion could lower the debris concentration in the narrow gap ${ }^{[4]}$. Given many available models, it appears that debris movement in EDM is not yet fully understood. 
This paper developed a model to simulate the movement and distribution of debris when the electrode conducted self-adaptive movement and experiments were performed to indicate that the quantity of debris flushed away was limited and most debris in the gap aggregates at one position can result in poor machining quality.

\section{EDM Mathematical Model}

A metal removal process with EDM is shown in Fig. 1. EDM uses the electro thermal effect of pulsed spark discharge between two electrodes (tool and workpiece) to remove materials in a dielectric fluid. The pulse energy, through discharges exerts on the local surface of both electrodes with dielectric fluid between them. In the narrow region of discharge gap the thermal effect of electrical current generates a plasma column with high temperature and high pressure, i.e., the discharging channel. The power supply instantaneously releases energy through discharging channel. Consequently, a small quantity of metal can be directly molten or even vaporized due to the heat effect, the molten metal is removed in the form of small spherical debris which is solidified by the liquid dielectric, leaving the discharging craters on both tool and workpiece surfaces.

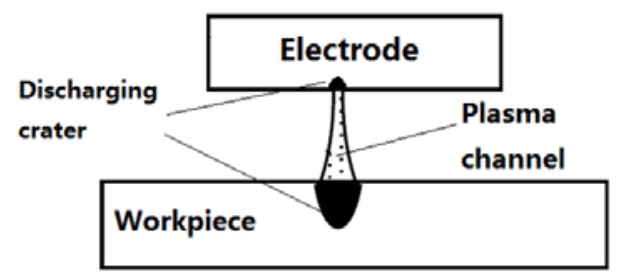

Fig. 1 Schematics of EDM principle

Inter-electrode Equation of Motion. Fluent predicts the trajectory of a discrete phase particle by integrating the force balance on the particle, which is written in a Lagrangian reference frame. This force balance equates the particle inertia with the forces acting on the particle, and can be written as

$\frac{d \mu_{p}}{d t}=F_{D}\left(\vec{\mu}-\vec{\mu}_{p}\right)+\frac{g\left(\rho_{p}-\rho\right)}{\rho_{p}}+\vec{F}$

where, $\vec{F}$ is an additional acceleration (force/unit particle mass) term, $F_{D}\left(\vec{\mu}-\vec{\mu}_{p}\right)$ is the $\operatorname{drag}$ force per unit particle mass and

$F_{D}=\frac{18 \mu C_{D} \operatorname{Re}}{24 \rho_{p} d_{p}^{2}}$

where, $\vec{\mu}$ is the fluid phase velocity, $\vec{\mu}_{p}$ is the particle velocity, $\mu$ is the molecular viscosity of the fluid, $\rho$ is the fluid density, $\rho_{\rho}$ is the density of the particle, and $d_{\rho}$ is the particle diameter. Re is the relative Reynolds number, which is defined as

$\operatorname{Re} \equiv \frac{\rho d_{p}\left|\vec{\mu}_{p}-\vec{\mu}\right|}{\mu}$

For the spherical particles, drag coefficient can be expressed as

$C_{D}=a_{1}+\frac{a_{2}}{\operatorname{Re}}+\frac{a_{3}}{\operatorname{Re}^{2}}$ 
where $a_{1}, a_{2}, a_{3}$, are constants.

Modeling for the Inter-electrode Gap Field. The side gap in EDM was set as $0.2 \mathrm{~mm}$ and bottom gap as $0.1 \mathrm{~mm}$. The debris generated in a single pulse was approximately considered in a uniform distribution at the bottom ${ }^{[5]}$.

The electrode was considered as a cylinder simplified as a 2D model which was established and meshed in the Gambit as shown in Fig. 2 (right). The tube and working liquid needed to exchange data so that an interface boundary condition was considered. The upper boundary condition was set to pressure outlet and rest boundaries were set to walls as default. The model was imported into Fluent. There're three layers of debris and distance between each layer is $0.05 \mathrm{~mm}$ and then a series of simulations were conducted. As the shape of flow field was constantly changing in response to the pulsed movement of the tool, the secondary development interface UDF module was utilized to simulate the process. Discharge experiments were conducted by using the machining parameters shown in Table 1.
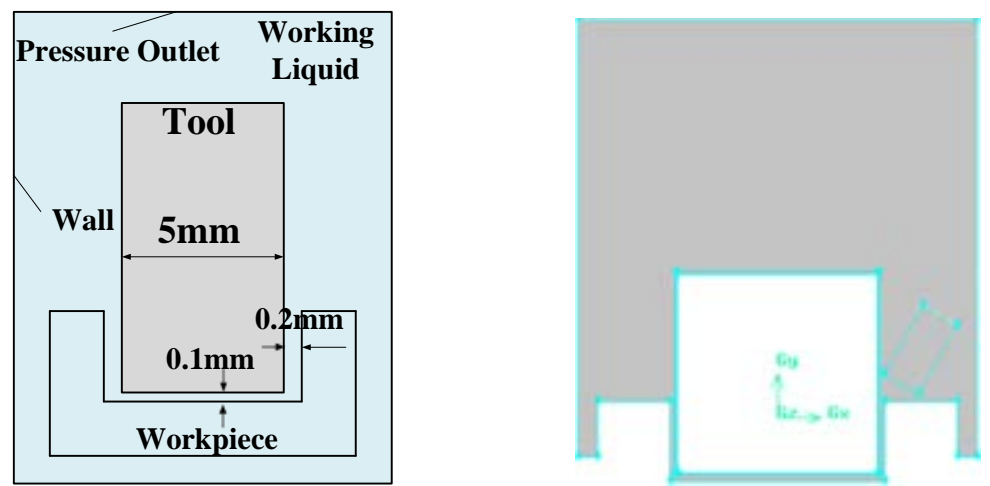

Fig. 2 the schematics of boundary conditions (left) and schematics of meshed model (right)

Table 1 Single discharge experimental parameters

\begin{tabular}{cccccccc}
\hline $\begin{array}{c}\text { Maintaining } \\
\text { voltage }\end{array}$ & $\begin{array}{c}\text { Peak } \\
\text { current }\end{array}$ & $\begin{array}{c}\text { Dielectric } \\
\text { fluid }\end{array}$ & $\begin{array}{c}\text { Flushing } \\
\text { velocity }\end{array}$ & Tool & Workpiece & $\begin{array}{c}\text { Tool } \\
\text { velocity }\end{array}$ & $\begin{array}{c}\text { Tool } \\
\text { Diameter }\end{array}$ \\
\hline $15 \mathrm{~V}$ & $0.8 \mathrm{~A}$ & Deionized water & $0.02 \mathrm{~m} / \mathrm{s}$ & Red Copper & TC4 & $0.01 \mathrm{~m} / \mathrm{s}$ & $0.005 \mathrm{~m}$ \\
\hline
\end{tabular}

\section{Simulation Results and Analysis}

Impact of Tool Movement on Gap Flow Field. The servomechanism advances the electrode towards the workpiece as the operation progresses, senses the discharge space, controls it to make self-adaptive movement and maintains a proper gap which is necessary to a successful machining operation.

Gap Flow Field with 0.5mm Deep Hole. The simulation results are shown in Fig. 3. As the tool moves down into the shallow hole, a positive pressure zone forms and the highest pressure appears in the bottom (See Fig. 3(b)). The electrode movement can induce turbulence in the gap pushing dielectric and debris flowing to the surrounding areas. Plenty of debris is soon washed away, leaving little debris at the bottom gap (See Fig. 3(a)). In the velocity field (See Fig. 3(c)), the highest speed position appears at the edge of tool at $0.25 \mathrm{~m} / \mathrm{s}$. As the tool moves back, the pressure reduces and a negative zone generates. This upward movement enhances the cleaning by bringing fresh dielectric into the gap which lowers the debris concentration and it is benefit to the next discharge.

Gap Flow Field with $\mathbf{2 m m}$ Deep Hole. As shown in Fig. 4, the electrode moves down into $2 \mathrm{~mm}$ deep hole, as shown in Fig. 4(a) only little debris is washed away, most debris is left in the gap and the accumulative debris could result in secondary discharge, which is harmful to the next stage machining. Both pressure field and velocity field are similar to $0.5 \mathrm{~mm}$ deep field (See Fig. 4 (a)-(b)). However, the highest readings for velocity and pressure are greater than the field of $0.5 \mathrm{~mm}$. 
In the shallow hole, most debris is easy to be washed away and debris concentration at the bottom is low. In the deep hole, little debris is washed away from the gap by dielectric fluid leaving a lot at the gap.

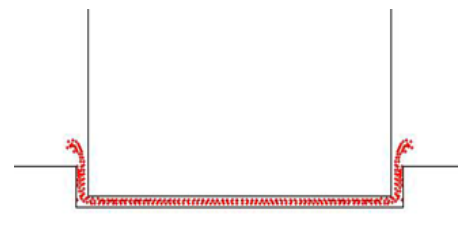

(a) debris distribution

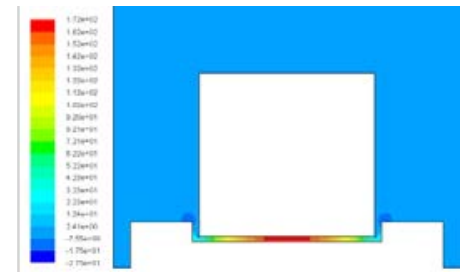

(b) pressure field

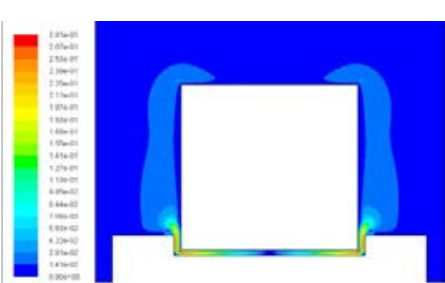

(c) velocity field

Fig. 3 the simulation results with $0.5 \mathrm{~mm}$ deep as tool moves down.

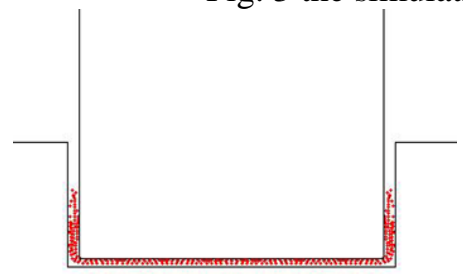

(a) debris distribution

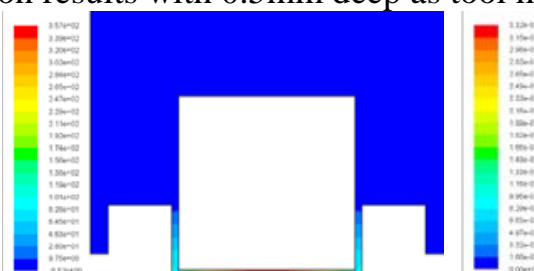

(b) pressure field

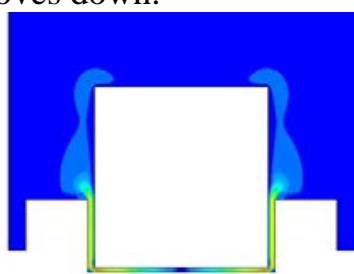

(c) velocity field

Fig. 4 the simulation results with $2 \mathrm{~mm}$ deep as tool moves down.

Impact of tool movement on gap flow field with flushing. The side flushing is added.

Gap Flow Field with 0.5mm Deep Hole. Fig. 5 shows the simulation results. In the machining process of $0.5 \mathrm{~mm}$ deep hole, as the tool moves towards the workpiece, a positive pressure zone is generated (See Fig. 5(d)), a high pressure region appears at the right gap where the liquid is colliding with the side flushing and deionized liquid as well as debris is driven to the surrounding areas by pressure difference (See Fig. 5(a)). Due to the shallow hole, most debris is flushed away from the gap. Considering the flushing impact, debris in the left gap is more than right one. A swirl is generated near the tube and the highest speed increases to $1.2 \mathrm{~m} / \mathrm{s}$. The speed of dielectric in the right side gap is $0.2 \mathrm{~m} / \mathrm{s}$ considering flushing influence, but the speed on the left is close to $0.3 \mathrm{~m} / \mathrm{s}$, the liquid reaches the highest speed at $0.4 \mathrm{~m} / \mathrm{s}$ at the lower left corner. Afterwards the tool moves up, a negative pressure zone forms which is less than surrounding pressures simultaneously (See Fig.5 (e)). Debris driven by the pressures of fluid moves towards the center. Due to the flushing influence, debris is flushed to the lower left position (See Fig. 5(c)). And the speed reduces to $0.9 \mathrm{~m} / \mathrm{s}$.

Gap Flow Field with $2 \mathrm{~mm}$ Deep Hole. Fig. 6 shows the simulation results. In the process of the tool moving down, the pressure increases as a positive pressure zone forms, dielectric liquid is washed to the surrounding areas, but colliding with flushing, pressure on the right side is higher than the left. As the tool arrives at the lowest position, the highest pressure appears at the bottom gap, dielectric liquid is diffused to different directions, flushing is hardly injected into the gap surrounding at the inlet where the pressure reaches the highest value (See Fig. 6(a) (d)). The highest speed is $0.4 \mathrm{~m} / \mathrm{s}$ in the gap as the tool reaches to the lowest position. As the tool moves back, the pressure reduces and a negative pressure field forms. Flushing brings fresh dielectric liquid into the gap and cools the tool and workpiece. The deeper the cavity, the greater the difficulty for proper flushing flowing through the gap so that the debris is not easily washed away, only little is removed, leaving most debris at the lower left corner (See Fig. 6(c)). This phenomenon induces a secondary discharge leading to a low accuracy finally. The speed reduces after the tool jumps (See Fig. 6(i)).

In the shallow hole, most debris is washed to the left gap by flushing and therefore the flushing has a larger impact than the tool movement. Whereas in deep hole, flush can not be fully injected into the gap, the pumping effect is not obvious so that it has little impact on the debris motion. 


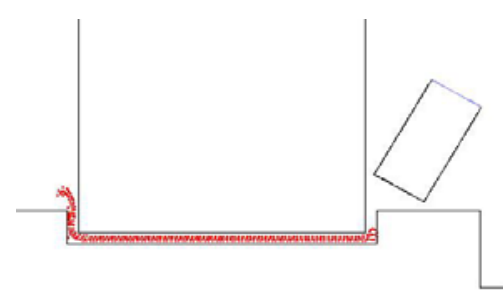

(a) downward movement

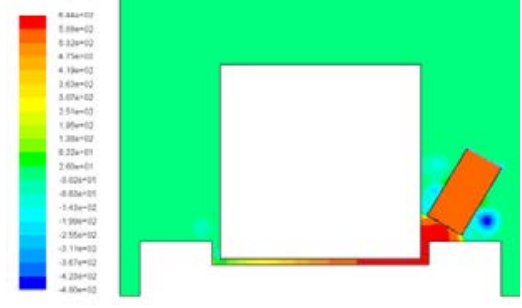

(d) downward movement

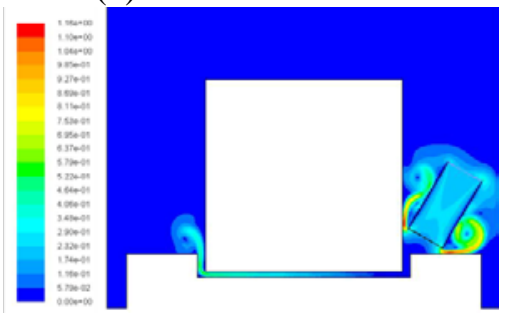

(g) downward movement

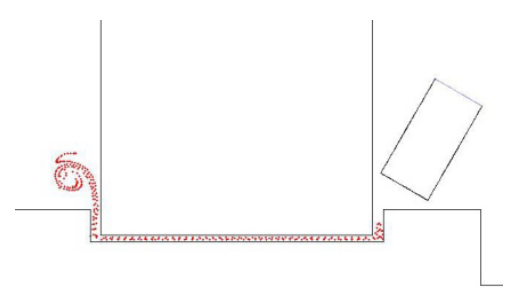

(b) beginning of moving up

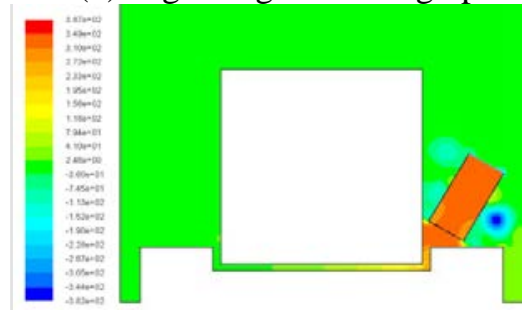

(e) beginning of moving up

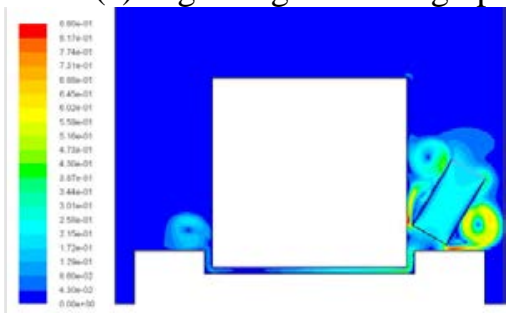

(h) beginning of moving up

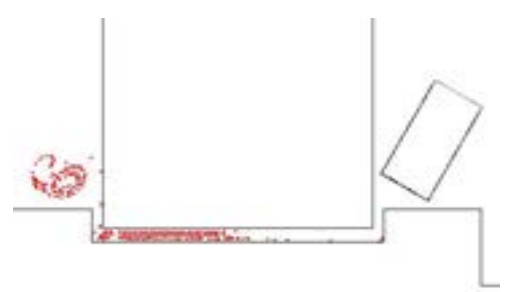

(c) upward movement

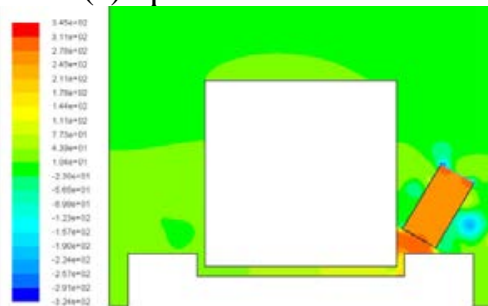

(f) upward movement

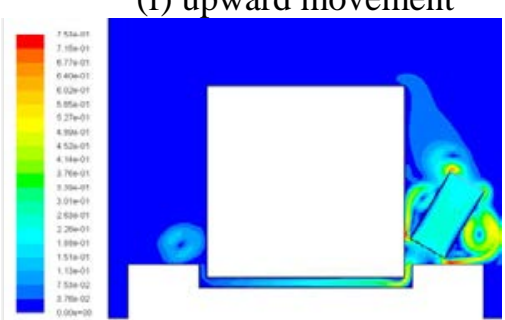

(i) upward movement

Fig. 5 the simulation results with $0.5 \mathrm{~mm}$ deep hole

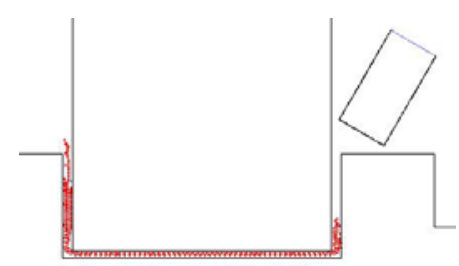

(a) downward movement

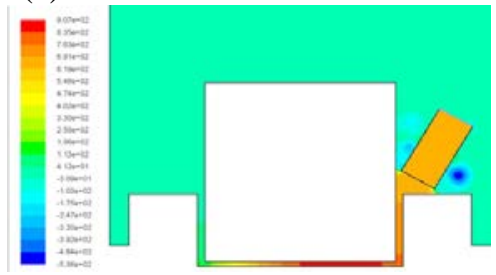

(d) downward movement

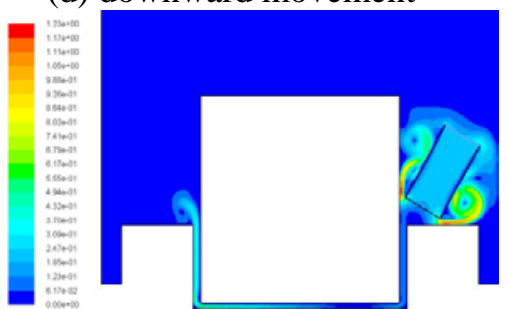

(g) downward movement

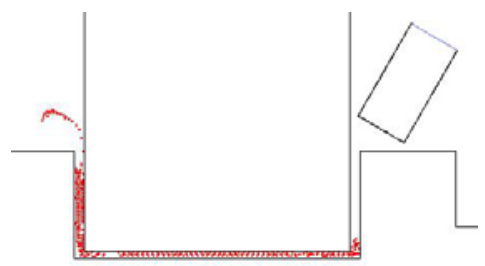

(b) beginning of moving up

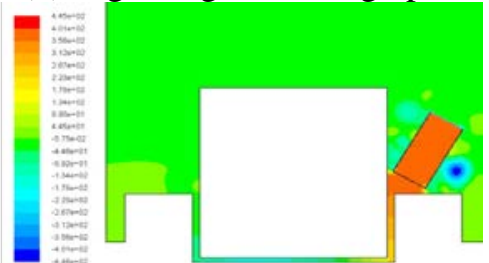

(e) beginning of moving up
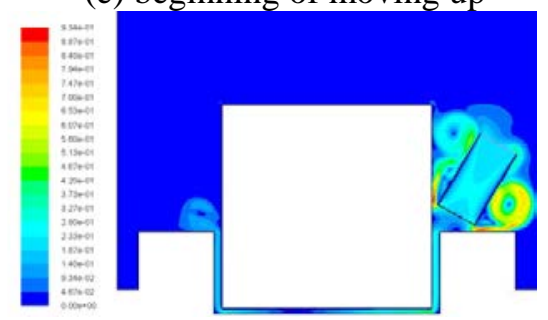

(h) beginning of moving up

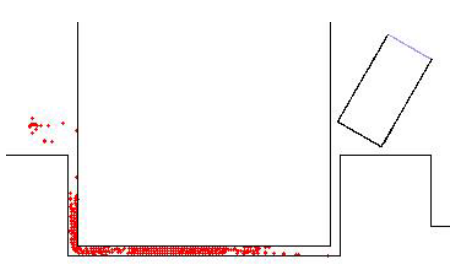

(c) upward movement
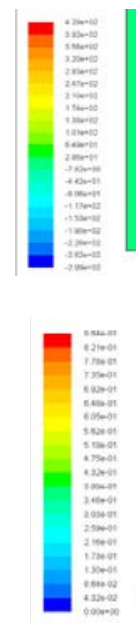

(i) upward movement
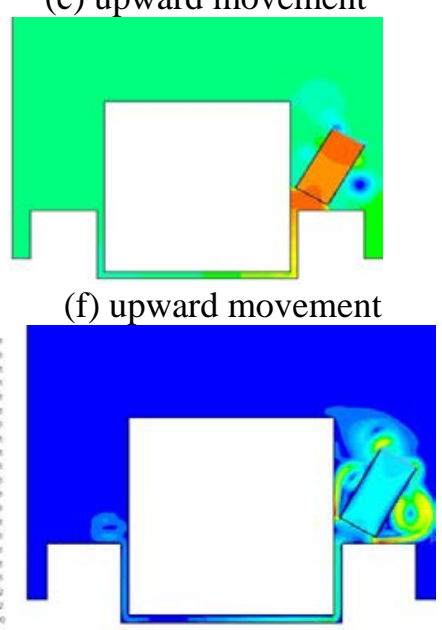

(f) upward movement Fig. 6 the simulation results with $2 \mathrm{~mm}$ deep hole

\section{Experiment Results and Discussion}

The experiments were carried out on a self-made machine. The parameter we applied was same to Table 1 . The tool initially moved down and made self-adaptive movement to maintain a proper spark-gap between tool and workpiece. The process was not terminated until a spark was seen under the bottom of workpiece. The cavity was $2 \mathrm{~mm}$ deep. An electron microscope was utilized to observe the surface appearance for both tool and workpiece. 
Fig. 7(a) shows the surface appearance after the machining process. As the electrode moved up and down in the cavity, this up and down motion caused a pumping action which drew in fresh dielectric liquid leading to the movement of debris. Due to the liquid impact, the debris was washed away uniformly from both sides, therefore, the bottom of electrode appeared approximately even.

In the jet flushing process, most debris stayed at the lower left corner, the accumulated debris can reduce electrical conductivity of the dielectric and a secondary discharge is easily created before the energy are fully reloaded. This procedure can over wear the electrode seriously, even leaving a slope appearance, as shown in Fig. 7(b). As the machining progressed, plasma channel could be created in the gap where few debris stayed at the right corner, at last materials were worn out and no longer existed. After several successive discharges, the workpiece surface appeared a shape with left higher than the right as shown in Fig. 7(c).

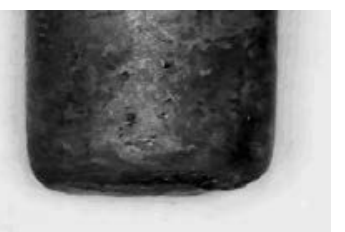

(a) tool without flushing

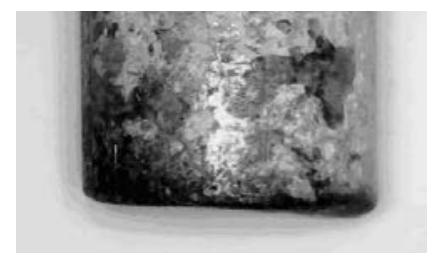

(b) tool with flushing

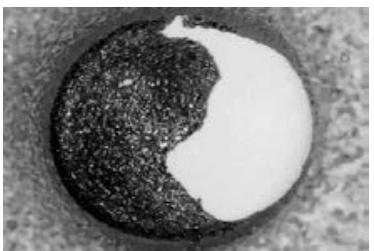

(c) hole with flushing

Fig. 7 Surface appearance after machining

\section{Conclusions}

Flushing can influence the motion of debris in EDM. A model was developed and a comparison was conducted to study the laws of debris movement and gap flow field. Relative Experiments were also carried out on a self-made machine to verify the model and conclusions were obtained as: 1 ) Debris removal normally is not a problem in a shallow machining process because there is sufficient pressure for the dielectric to flow through. Debris removal problems usually occur during deep operations. The deeper gap makes it more difficult to achieve the proper dielectric flow to remove the eroded debris, so higher pressure is required. 2) Eroded debris can easily cause secondary discharge when aggregate at the lower corner on the opposite of flushing direction and uneven wear can be generated under the bottom of electrode leaving poor machining quality. 3) The modeling using Fluent software is proven feasible by comparing with experiment.

\section{Acknowledgments}

The financial support from the National Natural Science Foundation of China under grant number 51405058, Doctoral Scientific Research Foundation of Liaoning Province under grant number 20131013 and Doctoral Program Foundation of Chinese Ministry of Education under grant number 20122124120001 are acknowledged.

\section{References}

[1]. B.B. Pradhan. Investigation of Electro-Discharge Micro-Machining of titanium super alloy. Int J Adv Manuf Technol. Vol. 41 (2009) No. 11, p. 1094-1106.

[2]. Antoine: Characterization of Electrical Discharge Machining Plasmas (Doctor, Lausanne, EPFL 2005). p.4.

[3]. Z.Y. Jia. X.Y. Zheng: Research on Flow State of Inter-Electrode Working Fluid in Micro-EDM. Journal of Dalian University of Technology. Vol. 50 (2013) No. 2, p. 188-193.

[4]. Z.L. Chu: Study of Electrode Jump Motion and EDM Performance (Graduate, Shanghai JiaoTongUniversity, China 2013). p.41.

[5]. J.Wang: Study on Debris and Bubble Movement and Control Strategy for Realizing High Efficiency Machining of Electrical Discharge Machining (Doctor, Dalian University of Technology, China 2012). p.39. 